\title{
Predictive role of skin rash in advanced pancreatic cancer patients treated with gemcitabine plus erlotinib: a systematic review and meta-analysis
}

This article was published in the following Dove Press journal:

OncoTargets and Therapy

\author{
Minyan Zengl,* \\ Qi Fengl,* \\ Ming Lu ${ }^{2}$ \\ Jun Zhou ${ }^{2}$ \\ Zuyao Yang ${ }^{1,3}$ \\ Jinling Tang ${ }^{1,3}$
}

'Division of Epidemiology, JC School of Public Health and Primary Care, The Chinese University of Hong Kong, Hong Kong SAR, China; ${ }^{2}$ Key Laboratory of Carcinogenesis and Translational Research (Ministry of Education), Department of GI Medical Oncology, Peking University Cancer Hospital \& Institute, Beijing, China; ${ }^{3}$ Cochrane Hong Kong, JC School of Public Health and Primary Care, The Chinese University of Hong Kong, Hong Kong SAR, China

*These authors contributed equally to this work
Correspondence: Zuyao Yang Division of Epidemiology, JC School of Public Health and Primary Care, The Chinese University of Hong Kong, 30-32 Ngan Shing Street, Hong Kong SAR, China

Tel +852 22528739

Fax +852 26063500

Email zyang@cuhk.edu.hk
Purpose: The survival benefit from gemcitabine plus erlotinib was on average marginal for advanced pancreatic cancer (APC) patients. Skin rash developed shortly after starting treatment seemed to be associated with better efficacy and might be used to assist clinical decision-making, but the results across studies were inconsistent. Thus, we conducted a systematic review and meta-analysis.

Methods: PubMed, Embase, Cochrane Central Register of Controlled Trials, three Chinese databases, and the abstracts of important conferences were searched for eligible studies. The primary outcome was overall survival (OS), and the secondary outcomes were progression-free survival (PFS) and objective response. The random-effects model was used to pool results across studies if heterogeneity was substantial. Otherwise, the fixed-effect model was used.

Results: A total of 16 studies with 1,776 patients were included. Patients who developed skin rash during treatment had longer OS (8.9 vs 4.9 months, HR=0.57, 95\% CI 0.50-0.64) and longer PFS (4.5 vs 2.4 months, HR=0.53, 95\% CI 0.40-0.68) than those who did not. A doseresponse relationship was also observed for both OS ( $\mathrm{HR}=0.64$ for grade- 1 rash vs no rash and $\mathrm{HR}=0.46$ for $\geq$ grade- 2 rash vs no rash) and PFS ( $\mathrm{HR}=0.72$ for grade- 1 rash vs no rash and $\mathrm{HR}=0.43$ for $\geq$ grade- 2 rash vs no rash).

Conclusion: Skin rash was associated with better OS and PFS in APC patients treated with gemcitabine plus erlotinib. It might be used as a marker for efficacy to guide clinical decisionmaking toward a more precise and personalized treatment.

Keywords: pancreatic neoplasms, targeted treatment, acne, prognosis

\section{Introduction}

As a highly malignant disease, pancreatic cancer is the leading cause of cancer-related deaths in the world, including the US, the UK, and Hong Kong. ${ }^{1-3}$ More than $80 \%$ of patients are diagnosed when the cancer is already at a locally advanced or metastatic stage, which is often referred to as advanced pancreatic cancer (APC). ${ }^{2}$ Without adequate treatment, the median survival of these patients is only $\sim 3-5$ months. ${ }^{4}$ For years, gemcitabine has been the standard treatment of APC. ${ }^{5}$ However, its benefit is small, with an increase of only 1.2 months in overall survival (OS). ${ }^{6}$ Recently, three regimens, namely gemcitabine plus erlotinib (6.24 vs 5.91 months, $P=0.038){ }^{7}$ gemcitabine plus nab-paclitaxel (8.5 vs 6.7 months, $P<0.001){ }^{8}$ and combination therapy of oxaliplatin, irinotecan, fluorouracil, and leucovorin (FOLFIRINOX; 11.1 vs 6.8 months, $P<0.001),{ }^{9}$ were proved to be able to achieve longer survival as compared with gemcitabine alone. 
Although the survival benefit provided by gemcitabine plus erlotinib is less than that by gemcitabine plus nabpaclitaxel or FOLFIRINOX, this regimen is associated with much fewer severe (grade 3 or grade 4) adverse events as compared with the other two regimens. ${ }^{7-9}$ In fact, the adverse events induced by gemcitabine plus erlotinib are mostly mild or moderate (grade 1 or grade 2). ${ }^{7}$ Interestingly and more importantly, it seems that those who are thus treated and develop skin rash during the treatment could achieve a significantly longer survival than those without skin rash. For example, Aranda et $\mathrm{al}^{10}$ found that the median survival with gemcitabine plus erlotinib was 3.3, 6.6, and 10.3 months in patients who developed no rash, grade- 1 rash, and $\geq$ grade- 2 rash, respectively $(P<0.001)$. Similar results were shown by Moore et al. ${ }^{7}$ In the study of Beveridge et al, ${ }^{11}$ the benefit was even larger (5.2 vs 12.0 months for rash vs no rash, $P=0.025$ ). This relationship was also observed in patients with other cancers treated with erlotinib. ${ }^{12,13}$ These findings suggested that skin rash could be a useful marker for predicting the efficacy of gemcitabine plus erlotinib and informing treatment decision.

However, the findings of existing studies regarding the role of skin rash seem inconsistent. For example, in some studies, the median survival time of patients with rash was longer than that of patients without rash, ${ }^{11}$ while in others, it was the other way round. ${ }^{14}$ Some suggested that rash could be a useful marker to inform choice of treatment, ${ }^{7,10,11}$ while others disagreed and even stated that "decision for interruption or maintenance of GEM + E, therefore, should not be based on the rash phenomenon". ${ }^{14,15}$ Importantly, many studies had small sample size, and their results were statistically insignificant. ${ }^{14-20}$ Could the discrepancy between the studies be explained merely by chance or different sample sizes? Alternatively, could effect modifiers such as some clinical characteristics play important roles, so that skin rash is truly not associated with prolonged survival in some populations? The answer is yet to be explored. Thus, we conducted a systematic review and meta-analysis to synthesize existing evidence on the association of skin rash with clinical outcomes in APC patients treated with gemcitabine plus erlotinib.

\section{Materials and methods}

\section{Data sources and literature search}

We performed a systematic search of PubMed, Embase, Cochrane Central Register of Controlled Trials, China Biology Medicine (in Chinese), Wanfang Data (in Chinese), and the China National Knowledge Infrastructure (in Chinese) from inception through 16 August 2017 using the following key words and their synonyms or their Chinese counterparts: pancrea*, cancer*, carcinoma*, adenocarcinoma*, tyrosine kinase inhibitor*, erlotinib, tarceva, OSI-774, and OSI774. As analysis on the relationship between skin rash and clinical outcomes might not be the major interest of some potentially eligible studies, the terms related to skin rash may not appear in their searchable fields, and thus, we did not use those terms in literature search. There were no restrictions on language or publication status (abstracts and full text) of studies. Wherever possible, the searches were limited to "human studies". To supplement the search of electronic databases, the meeting abstracts of American Society of Clinical Oncology and European Society of Medical Oncology were reviewed manually.

\section{Study selection}

Two reviewers (MZ and QF) screened the records' titles and abstracts independently to judge their relevance to this systematic review. Full texts of the studies that appeared to meet the inclusion criteria listed earlier were obtained for further examination. Potentially eligible studies selected by the two reviewers were then combined and discussed for final decision on their eligibility. Studies that fulfilled all the following criteria were considered as eligible for this systematic review: 1) study participants were patients diagnosed with APC; 2) the patients were treated with gemcitabine plus erlotinib; 3 ) at least one of the following clinical outcomes were assessed: OS (the time from start of treatment to death or loss of follow-up, whichever occurred first), progression-free survival (PFS; the time from start of treatment to radiological progression or loss of follow-up, whichever occurred first), and objective response assessed according to Response Evaluation Criteria In Solid Tumors or its updated versions; 4) skin rash was assessed according to National Cancer Institute Common Toxicity Criteria (NCI-CTCAE), its updated versions, or others (such as WHO Toxicity Criteria [WHO-TC]); and 5) the relationship between skin rash and clinical outcomes was assessed. The reference lists of eligible studies and relevant reviews were also scrutinized for additional eligible studies. If two or more reports were available from one study, either some of them were excluded or they were combined to get a full picture of the study, depending on the extent of duplication (complete or partial).

\section{Data extraction}

After eligible studies were identified, the following data were extracted from the study: 1) bibliographic information, such 
as the first author, country, and publication year; 2) baseline clinical characteristics of patients, such as the number of patients with rash and those without, age, sex, Eastern Cooperative Oncology Group performance status (ECOG PS) level, pathological type and treatment; 3) main numerical results, such as HRs and 95\% CIs for OS and PFS, ORs and 95\% CIs for objective response, and other data from which the effect estimates could be calculated; and 4) information on methodological quality (see in the following).

Authors of original studies were contacted as needed to clarify ambiguities in reported methods or results and to seek additional data omitted in the publications. If not explicitly reported in original publications and still not available after contacting with the author, HRs were estimated using the method developed by Parmar et al, ${ }^{21}$ which was recommended by the Cochrane Handbook for Systematic Reviews. ${ }^{21}$ Data were extracted independently by two reviewers ( $\mathrm{MZ}$ and $\mathrm{QF}$ ) using a standardized data extraction form. Disagreements between the two reviewers were resolved by revisiting original publications and discussion until consensus was reached.

\section{Risk of bias assessment}

All eligible studies could be viewed as cohort studies in terms of their methodological rigor, as the comparison of rash with no rash was and could only be observational. Since no gold standard was available for methodological quality assessment of cohort studies, Newcastle-Ottawa Scale $^{22}$ was adopted in the present systematic review, which has been frequently used in other systematic reviews. This scale focuses on three study aspects, including selection of patients, comparability of baseline characteristics between comparison groups, and outcome assessment. For each aspect, there were up to four items for detailed evaluation. The overall study quality was denoted by a numerical score ranging from 0 to 9 , with 7-9 scores indicating good quality. Quality assessment was performed independently by two reviewers (MZ and QF). Disagreements between the two were resolved by revisiting original papers and discussing. Unresolved disagreements were referred to a third expert researcher (ZY) for a final decision.

\section{Data synthesis and analysis}

The primary clinical outcome of this study was OS, and the secondary outcomes included PFS and objective response. The clinical outcomes were compared between patients with rash vs those without and between patients with high-grade ( $\geq$ grade 2 ) rash vs those with low-grade rash ( $\leq$ grade 1).
The difference in OS or PFS between groups was measured by HR with $95 \% \mathrm{CI}$, and the difference in objective response was measured by OR with $95 \%$ CI. HRs and ORs from relevant studies were combined to produce a summary HR and OR, respectively. HR $<1$ or OR $>1$ meant the outcomes of those with skin rash during treatment were better than those without, while HR $>1$ or OR $<1$ meant the opposite. To provide clinicians and patients with more intuitive and straightforward information for their decision-making, we also synthesized the median survival times for the rash (and high-grade rash) group and the no-rash (and low-grade rash) one, respectively, using the method proposed by Zang et al. ${ }^{23}$ We also investigated dose-response relationship, the phenomenon that the probability of outcome (ie, OS and PFS in this systematic review) increases or decreases with the dose/level of exposure (ie, different grades of rash in this systematic review) by comparing the pooled effect estimate of grade-1 skin rash vs no rash with the pooled effect estimate of $\geq$ grade 2 skin rash vs no rash.

Statistical heterogeneity among studies was assessed by the Cochran's $Q$ test and the $I^{2}$ statistic. ${ }^{24} \mathrm{~A} P$-value $\leq 0.10$ for the $Q$ test or an $I^{2}$ value $>50 \%$ was suggestive of significant heterogeneity. If there was no significant heterogeneity, data from different studies will be pooled with the fixed-effects model. Otherwise, the random-effects model was used for pooling results and subgroup analyses were used to investigate potential sources of heterogeneity according to selected clinical factors such as ethnicity, performance status, and prior chemotherapy treatment for pancreatic cancer. If suitable data on the time from starting treatment to onset of skin rash were available, subgroup analysis according to the time would be conducted. Sensitivity analyses were conducted by excluding the studies according to two aspects, including methodological (eg, excluding the studies with low-quality score) and clinical characteristics (eg, excluding the studies that recruited a small number of patients receiving an additional therapy during the treatment). Begg's funnel plot and Egger's test were used to examine publication bias..$^{25}$ However, the two tests were conducted only when a metaanalysis included more than 10 studies and no substantial heterogeneity was observed among the studies; otherwise, the tests would have limited statistical power and misleading results. ${ }^{25,26}$ In presence of an asymmetric funnel plot, the Duval and Tweedie nonparametric trim-and-fill method was used to adjust for the potential reporting bias and obtain an adjusted result of meta-analysis. ${ }^{27}$ The synthesis of median survival times was conducted using SAS 9.3 (SAS Institute Inc., Cary, NC, USA), and the other analyses were performed 
using STATA (version 11.0; StataCorp LP, College Station, TX, USA).

\section{Results}

As shown in Figure 1, 4,327 records were retrieved by literature search and 16 studies with 1,776 patients were finally included in this systematic review. ${ }^{10,11,14,18,28-39}$ The characteristics of eligible studies are given in Table 1. In all, 12 studies were prospective and four retrospective. Eight studies were performed predominantly in Caucasians and seven in Asians. A total of 12 studies assessed

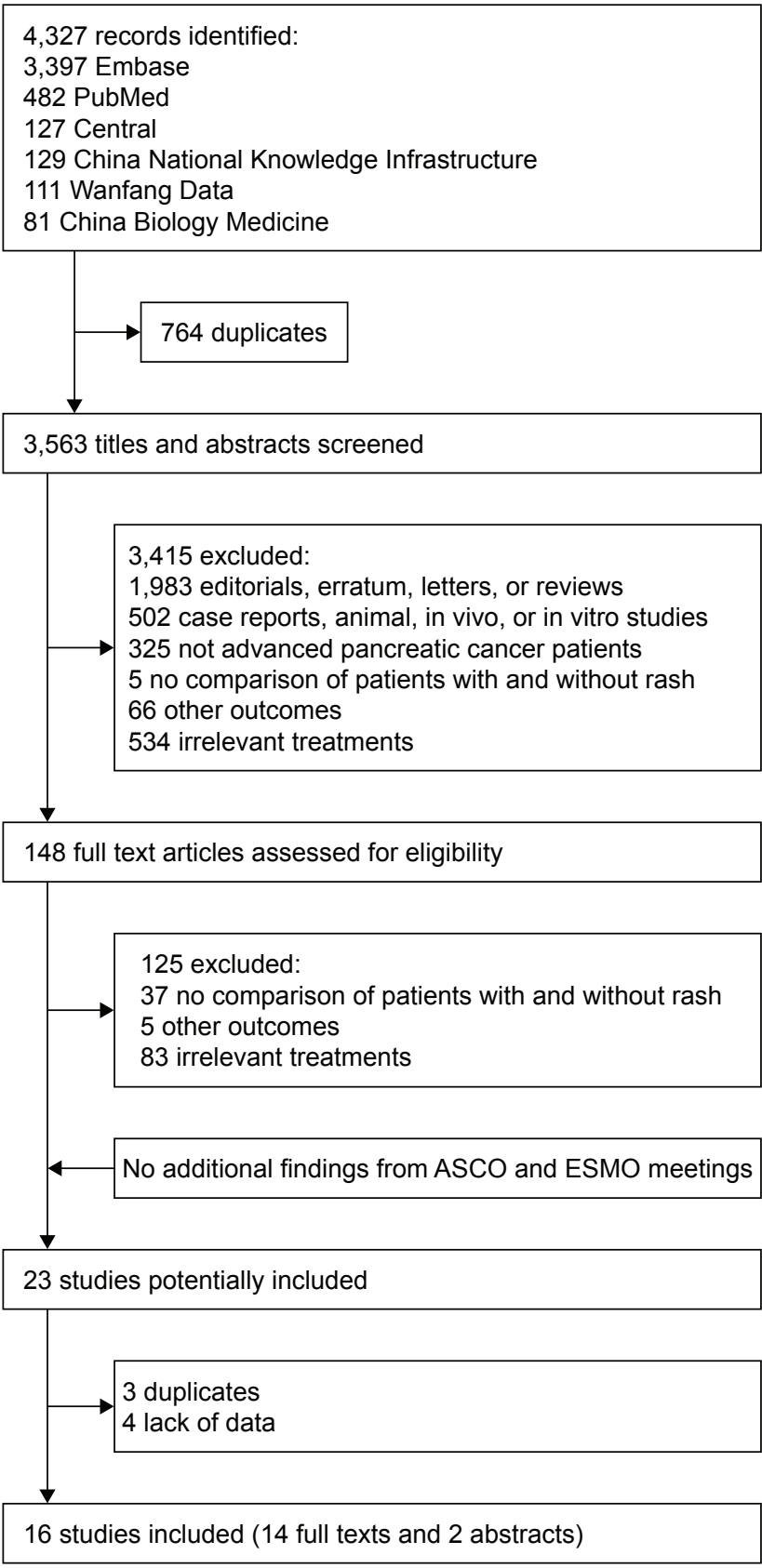

Figure I Flowchart of study selection.

Abbreviations: ASCO, American Society of Clinical Oncology; ESMO, European Society of Medical Oncology. skin rash according to the NCI-CTCAE and two according to the WHO-TC. The median/mean age reported by different studies ranged from 55.0 to 67.2 years (median: 63.9 years). The proportions of male patients ranged from $41.7 \%$ to $72.7 \%$ (median: $57.5 \%$ ). The proportions of patients with relatively good performance status (ECOG PS 0-1) ranged from $59.4 \%$ to $100 \%$ (median: $86.1 \%$ ). A total of 10 studies reporting histology enrolled only or mostly $(\geq 90 \%)$ patients with exocrine tumors. In 10 of the 12 studies reporting prior chemotherapy treatment, all or most $(>90 \%)$ of the patients were treatment naïve. Data on OS, PFS, and objective response were provided by 12 , nine, and eight studies, respectively. In all, 12 studies were assessed as with a low risk of bias (Table S1). In the process of data extraction and quality assessment, six disagreements occurred between the two reviewers (four in quality assessment and two in data extraction), but none of the disagreements was about the data of key results and all were resolved after discussion. Thus, they did not influence our main analyses.

\section{OS}

A total of 10 studies with 1,421 patients reported comparison of OS between the patients with rash and those without, and the pooled median OS time of them was 8.9 and 4.9 months, respectively $(\mathrm{HR}=0.57,95 \% \mathrm{CI} 0.50-0.64$, heterogeneity $I^{2}=23.3 \%, P=0.215$; Figure $2 \mathrm{~A}$ ). Four studies with 487 patients reported comparison between the patients with high- and low-grade rash ( $\mathrm{HR}=0.63,95 \%$ CI $0.49-0.80$, heterogeneity $I^{2}=0.0 \%, P=0.525$; Figure $\left.2 \mathrm{~B}\right)$. As severity of rash increased, the association of rash with OS became stronger (grade-1 rash vs no rash: $\mathrm{HR}=0.64,95 \%$ CI $0.47-0.88$, heterogeneity $I^{2}=60.7 \%, P=0.054$; grade- 2 rash vs no rash: $\mathrm{HR}=0.46$, 95\% CI $0.37-0.57$, heterogeneity $I^{2}=0.0 \%$, $P=0.433)$.

\section{PFS}

Seven studies with 927 patients compared PFS between the patients with rash and those without, and the pooled median PFS of them was 4.5 and 2.4 months, respectively $(\mathrm{HR}=0.53$, $95 \%$ CI $0.40-0.68$, heterogeneity $I^{2}=62.9 \%, P=0.006$; Figure $3 \mathrm{~A})$. A similar trend was observed in the comparison between high- and low-grade rash $(\mathrm{HR}=0.59,95 \%$ CI $0.48-0.74$, heterogeneity $I^{2}=23.5 \%, P=0.270$; Figure $3 \mathrm{~B}$ ). As severity of rash increased, the association of rash with PFS became stronger (grade- 1 rash vs no rash: $H R=0.72$, 95\% CI $0.55-0.94$, heterogeneity $I^{2}=55.2 \%, P=0.082$; grade-2 rash vs no rash: $\mathrm{HR}=0.43 ; 95 \%$ CI $0.35-0.53$, heterogeneity $I^{2}=0.0 \%, P=0.781$ ). 


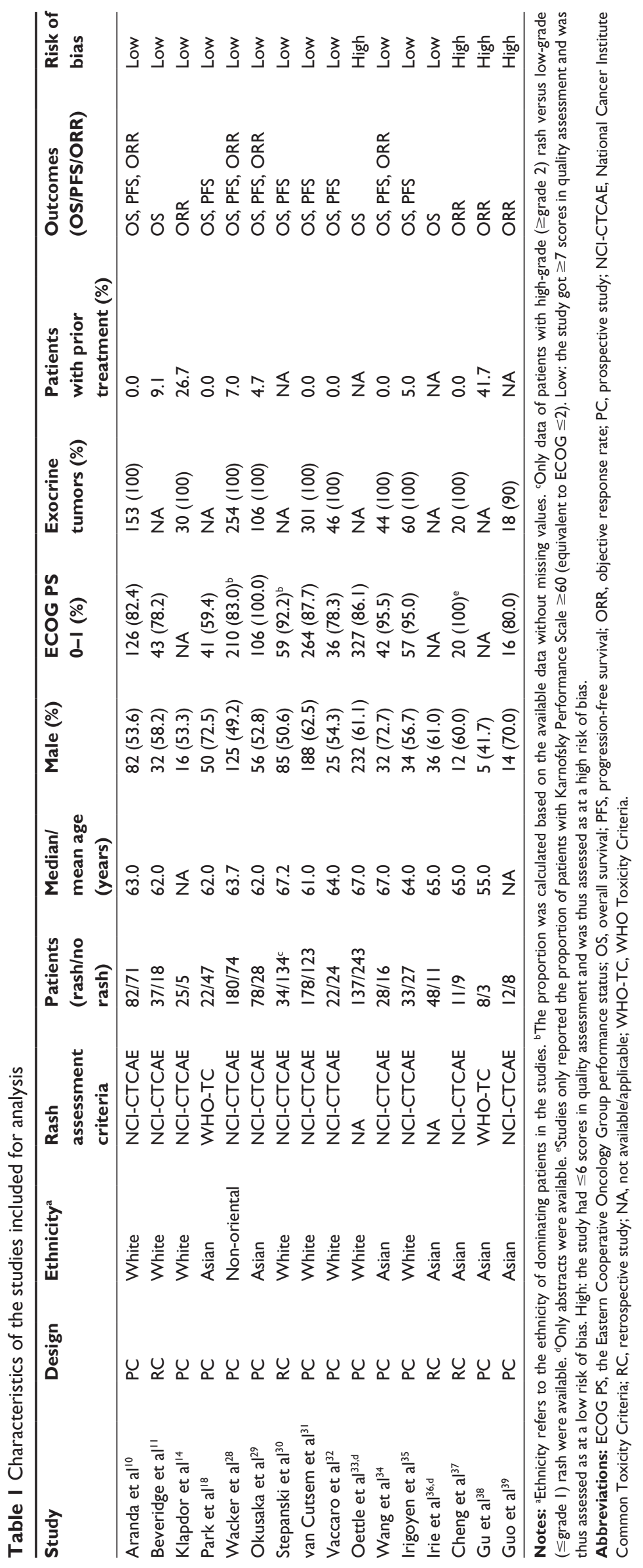


A Study ID

HR $(95 \% \mathrm{Cl})$

$\%$ weight

Wacker et al $(2007)^{28}(1)$

Wacker et al $(2007)^{28}(2)$

van Cutsem et al (2009) ${ }^{31}$

Vaccaro et al (2013) $)^{32}$

Park et al $(2013)^{18}$

Beveridge et al (2014) 11

Oettle et al (2013) ${ }^{33}$

Wang et al (2015) $)^{34}$

Aranda et al $(2012)^{10}(1)$

Aranda et al $(2012)^{10}(2)$

Irigoyen et al (2017) 35

Irie et al (2014)

Overall $\left(I^{2}=23.3 \%, P=0.215\right)$

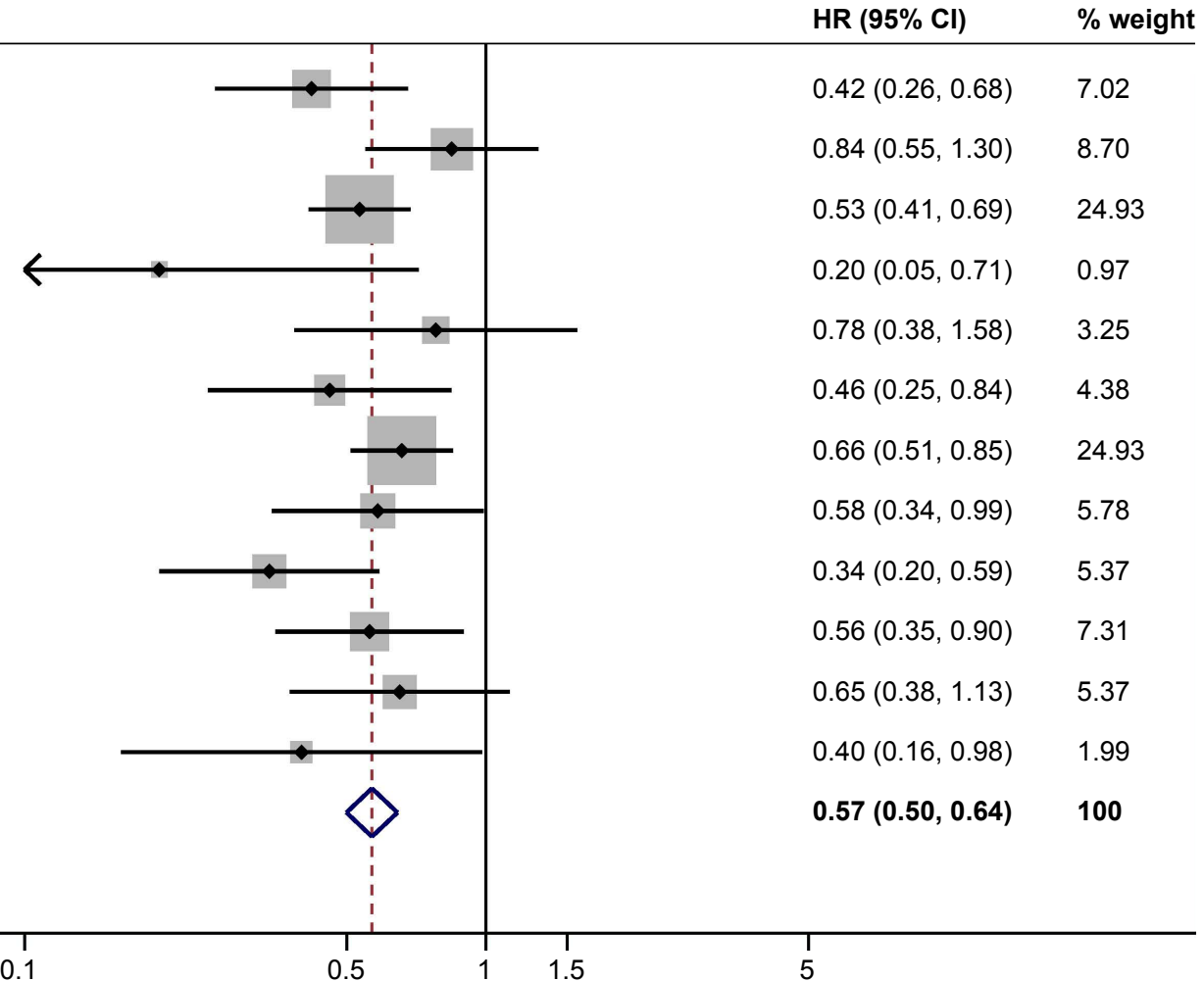

\section{B Study ID}

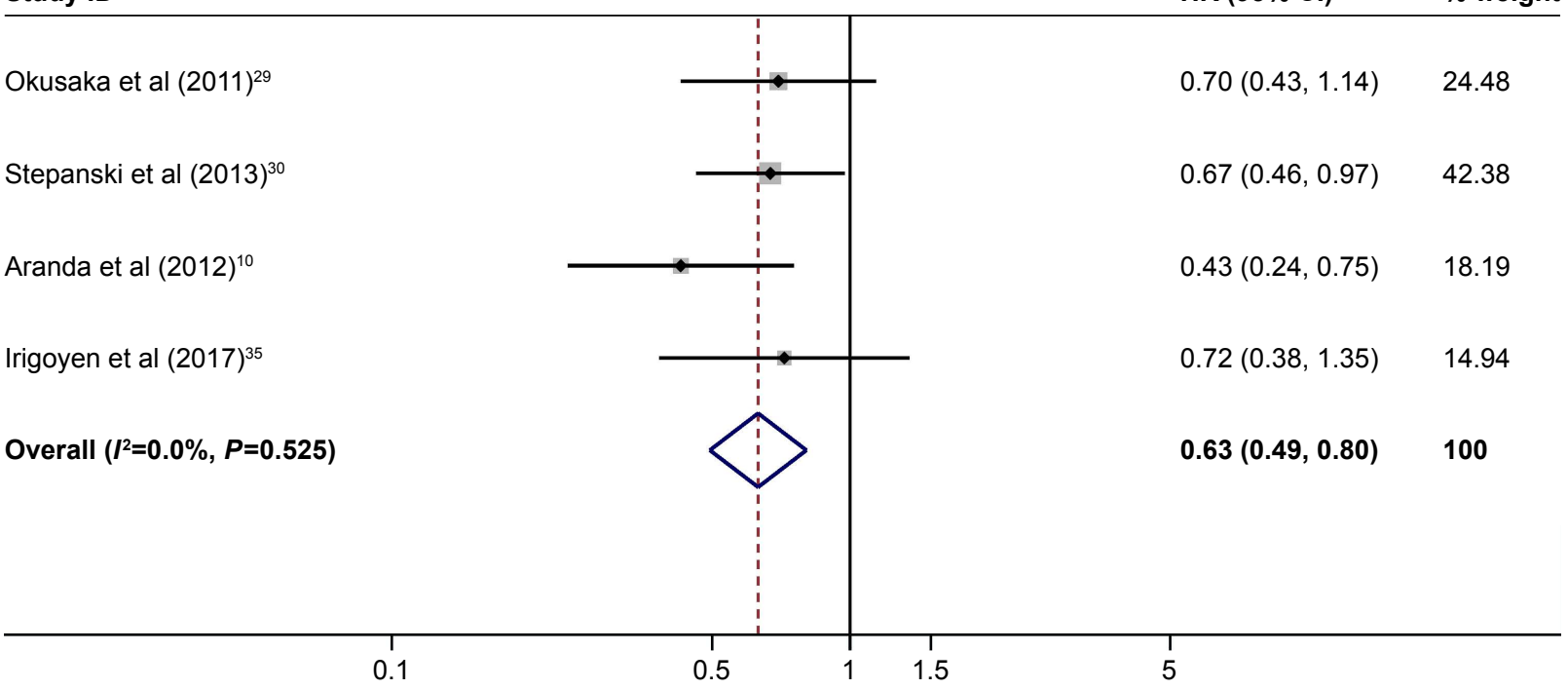

Figure 2 (A) Forest plot of HR for OS: with vs without rash. (B) Forest plot of HR for OS: high-grade rash vs low-grade rash.

Note: Both Wacker et al (2007) ${ }^{28}$ and Aranda et al $(2012)^{10}$ had two comparisons, because they reported the comparisons of grade-I rash with no rash and grade-2 rash with no rash separately, and the comparison of any-grade rash with no rash was not available.

Abbreviation: OS, overall survival.

\section{Objective response}

There was a trend that rash was associated with a higher likelihood of response in the comparison between rash and no $\operatorname{rash}(40 / 254$ [15.7\%] vs 9/107 [8.4\%], OR=1.87, 95\% CI $0.87-4.02$, heterogeneity $I^{2}=7.6 \%, P=0.363$; Figure 4A) and in the comparison between high- and low-grade rash $(39 / 202$ [19.3\%] vs $31 / 341$ [9.1\%], OR=2.20, 95\% CI
1.29-3.74, heterogeneity $I^{2}=0.0 \%, P=0.496$; Figure 4B), consistent with the results of OS and PFS.

\section{Sensitivity, subgroup, and publication bias analyses}

Sensitivity analyses according to methodological quality and clinical characteristics showed that the results described 


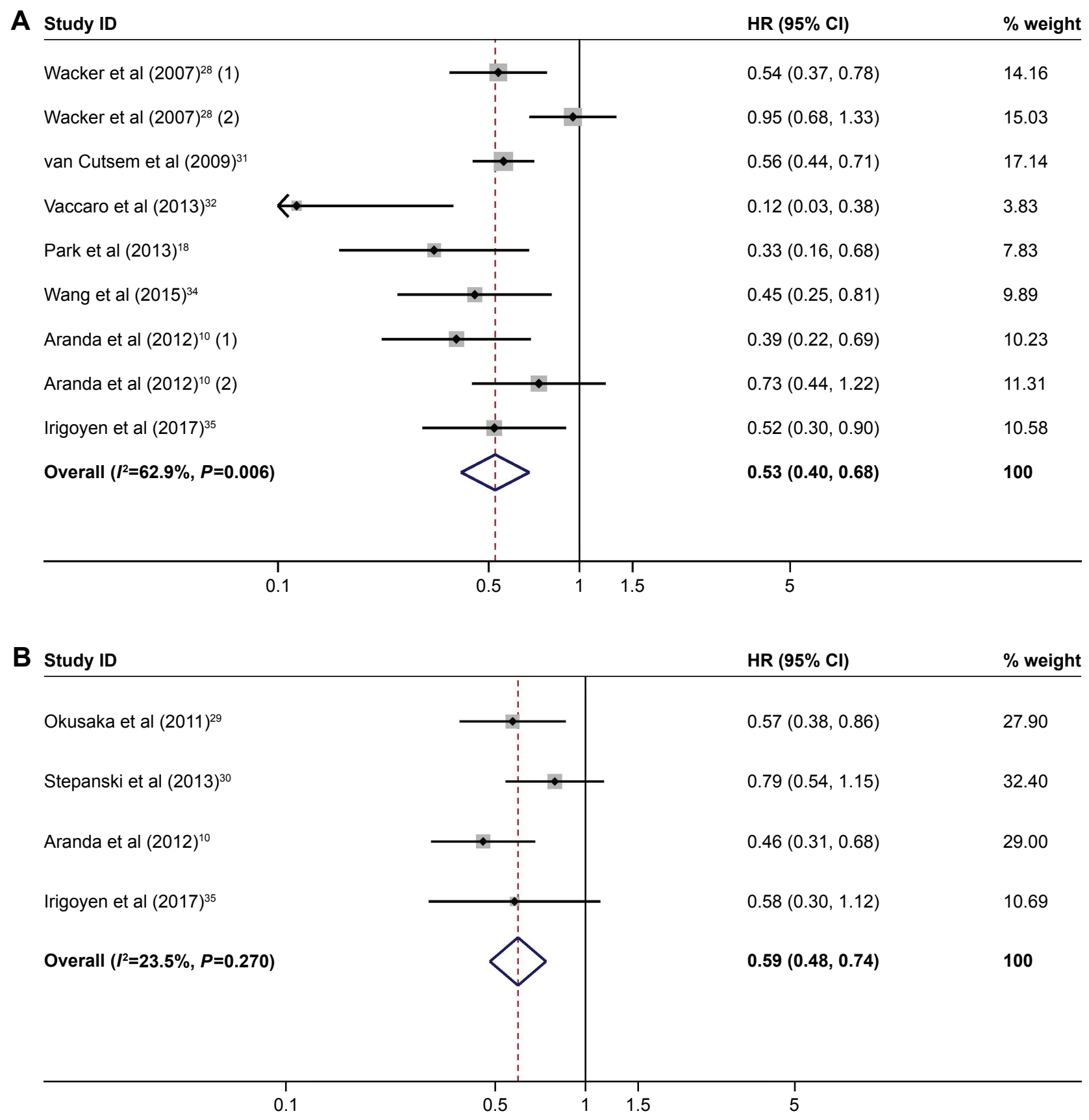

Figure 3 (A) Forest plot of HR for PFS: with vs without rash. (B) Forest plot of HR for PFS: high-grade vs low-grade rash.

Notes: Both Wacker et al $(2007)^{28}$ and Aranda et al $(2012)^{10}$ had two comparisons, because they reported the comparisons of grade-I rash with no rash and grade- 2 rash with no rash separately, and the comparison of any-grade rash with no rash was not available. Weights are from random-effects analysis.

Abbreviation: PFS, progression-free survival.

earlier were robust (Table S2). A series of prespecified subgroup analyses were conducted to investigate the potential source of substantial heterogeneity observed in Figure 3A. The results showed that ECOG physical performance status and prior chemotherapy treatment might be associated with the substantial heterogeneity (Table S3). Specifically, the associations between rash and clinical outcomes were stronger in the subgroup where more patients had worse performance status $(\geq 2)$ and in the subgroup where more patients were treatment naive. Only two studies ${ }^{10,28}$ included in our systematic review mentioned the time from starting treatment to onset of skin rash, but they merely reported the median time instead of individual data. Owing to limited data, we did not conduct subgroup analysis according to the time of onset of skin rash. Egger's tests for asymmetry of the funnel plot constructed based on the data of Figure 2A were not statistically significant ( $P=0.188$; Figure $\mathrm{S} 1$ ), suggesting no evidence for publication bias. Potential publication bias in 


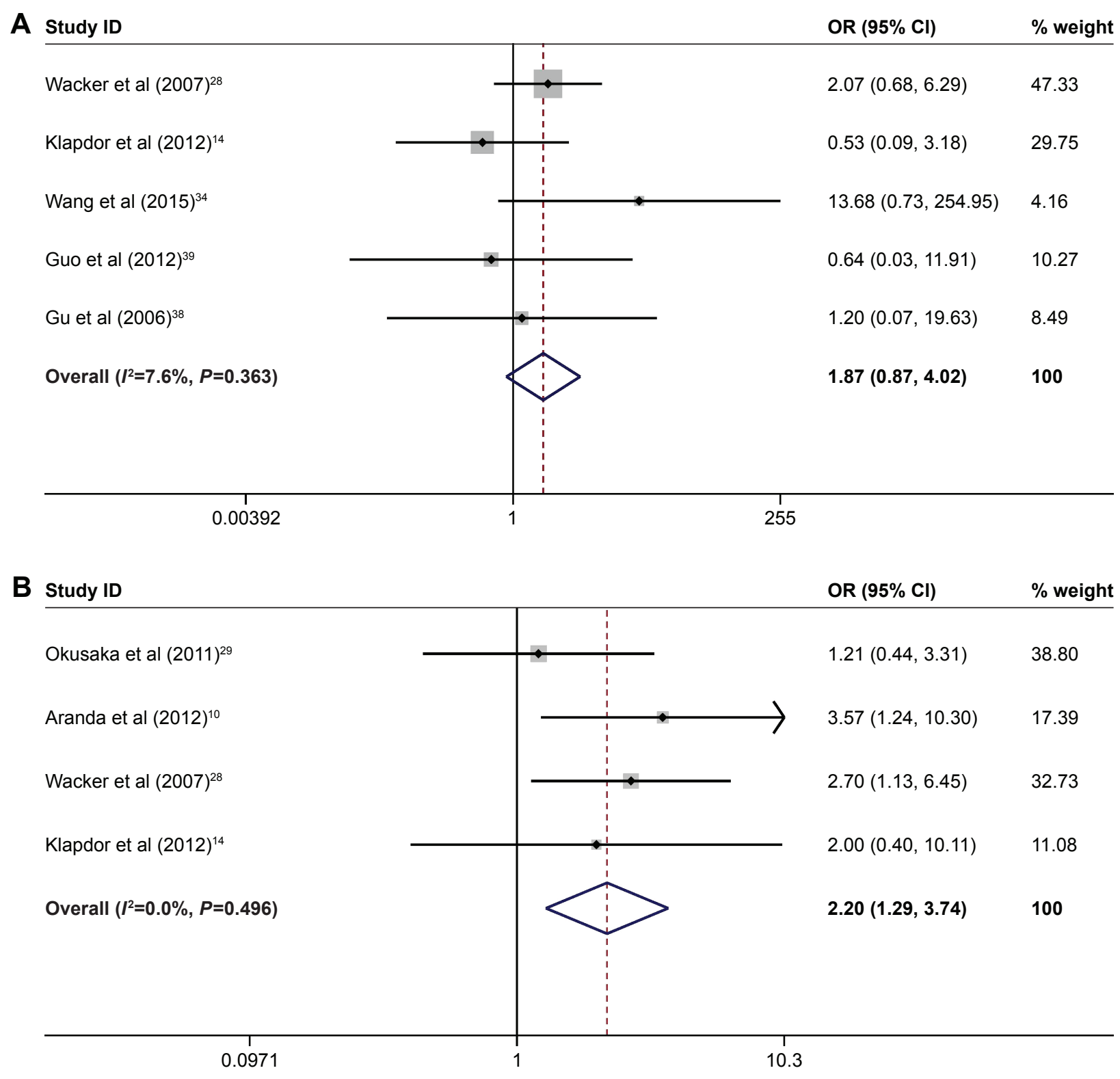

Figure 4 (A) Forest plot of OR for objective response: with vs without rash. (B) Forest plot of OR for objective response: high-grade rash vs low-grade rash. Note: Data analysis software automatically dropped Cheng et al $(2010)^{37}$ because of the zero events in both groups.

the data of other figures was not assessed due to the limited number of studies $(<10)$ included in these meta-analyses.

\section{Discussion}

This systematic review synthesized 16 studies with 1,776 patients and found that skin rash was significantly associated and had a dose-response relationship with longer OS and PFS in APC patients treated with gemcitabine plus erlotinib. Similar results were observed in cancers at other sites than pancreas. For example, the systematic reviews of Liu et $\mathrm{al}^{40}$ and Petrelli et al ${ }^{12,41}$ found that skin rash was associated with longer OS and PFS and higher objective response rate in lung cancer and colorectal cancer patients, respectively. A trial of erlotinib in patients with metastatic cancer of head and neck reported a dose-response relationship between skin rash and OS. ${ }^{13}$

However, it should be noted that the evidence summarized here is not the optimal for establishing the predictive role of skin rash, because it is well possible that skin rash was also associated with better outcomes in the gemcitabine-placebo arm, in which case the association was not specific to gemcitabine-erlotinib treatment, and thus, skin rash could not be used to predict the efficacy of the treatment. In epidemiology, the best design to evaluate 
whether a factor could predict treatment efficacy or not is randomized clinical trial with subgroup analysis according to the potential predictive factor and assessment of interaction between the factor and the treatment. ${ }^{42} \mathrm{We}$ did not conclude such evidence in the present systematic review because our pilot search showed that there was only one such trial, which was by Moore et al. ${ }^{7}$ Fortunately, Moore et $\mathrm{al}^{7}$ found that skin rash was significantly associated with longer OS (HR=0.74, 95\% CI 0.56-0.98, $P$-value $=0.037)$ in the gemcitabine-erlotinib arm but not in the gemcitabine-placebo arm $(\mathrm{HR}=0.90,95 \%$ CI $0.68-1.18$, $P$-value $=0.435),{ }^{28}$ suggesting that rash could predict the efficacy of gemcitabine-erlotinib treatment and thus supporting the findings of the present systematic review.

The association between skin rash and clinical outcomes may be explained by the following biological mechanisms. Erlotinib is a small molecule tyrosine kinase inhibitor of EGFR, which is frequently overexpressed in pancreatic cancer and associated with the progression of the disease. ${ }^{43}$ However, as EGFR is also expressed as undifferentiated and proliferating keratinocytes in the basal and suprabasal layers of the epidermis, the therapeutic effects of erlotinib may at the same time cause growth arrest and inflammation of epidermis that ultimately lead to skin rash. ${ }^{44,45}$ Patients who constantly have a high plasma drug concentration are more likely to develop skin rash. ${ }^{46}$ This is supported by two RCTs that reported that the appearance of rash was positively associated with the concentration of erlotinib. ${ }^{47,48}$ In fact, skin rash in the form of clustering pustular lesions on the face, neck, and upper body is the earliest, commonest and most characteristic adverse effect of EGFR-targeted drugs including erlotinib. ${ }^{7,12,49}$

Compared with objective response assessed by imaging techniques such as computed tomography (CT) and X-ray, ${ }^{50}$ skin rash is straightforward and easier to evaluate without introducing additional harms and costs. ${ }^{51}$ Furthermore, skin rash usually occurs within 8 days of starting the treatment, with maximal intensity in the second week, ${ }^{49}$ while the result of objective response assessment according to the Response Evaluation Criteria In Solid Tumors guidelines is usually not available until 6-8 weeks after starting treatment. ${ }^{50,52}$ Therefore, skin rash could be used to facilitate an early estimate of the efficacy of gemcitabine plus erlotinib and help clinicians and patients decide on whether to continue with the regimen or to switch to other regimens. These decisions may exempt patients suffering from unnecessary toxicity of a futile regimen, potentially save cost, and enable them to switch to other potentially effective treatments as early as possible. In addition to survival improvement, patients who develop rash during the treatment may also benefit psychologically from reassurance about the efficacy of treatment.

To our knowledge, this is the first systematic review looking at the relationship between skin rash and clinical outcomes of APC patients who received gemcitabine plus erlotinib. Most studies included had a low risk of bias. In addition, sensitivity analyses demonstrated that the results were robust.

However, still there are some limitations that need to be acknowledged. First, as mentioned earlier, we were unable to do publication bias analysis for some of the forest plots due to limited number of studies included. Second, though subgroup analyses revealed that the substantial heterogeneity observed in some meta-analyses was possibly caused by performance status and prior chemotherapy treatment for pancreatic cancer, caution should be taken when interpreting the results. This is because the cutoff points (proportion of patients with a specific characteristic) for defining subgroups were arbitrary, the subgroups were not distinct entities, and the number of studies was very small in some subgroups. Therefore, the possibility of false-positive results and ecological fallacy cannot be ruled out. Third, the statistical power of two studies included in our systematic review was limited and $95 \%$ CIs of effect estimates rather wide, because the two studies were very small in sample size. ${ }^{32,34}$ However, this is not necessarily a drawback, because meta-analysis is exactly meant to increase the statistical power of analysis and precision of results by synthesizing multiple studies with relatively small sample sizes.

\section{Conclusion}

Skin rash was associated with better clinical outcomes in APC patients treated with gemcitabine plus erlotinib. As skin rash occurs earlier and is more straightforward and easier to evaluate than other known markers without introducing additional harms and costs, it could be used to facilitate an early estimate of the efficacy of gemcitabine plus erlotinib and guide clinical decision-making toward a more precise and personalized treatment.

\section{Abbreviations}

APC, Advanced pancreatic cancer; FOLFIRINOX, combination therapy of oxaliplatin, irinotecan, fluorouracil, and leucovorin; NCI-CTCAE, National Cancer Institute Common Toxicity Criteria; WHO-TC, WHO Toxicity Criteria; ECOG PS, Eastern Cooperative Oncology Group performance status. 


\section{Acknowledgment}

This study was funded by Health and Medical Research Fund (Ref No 14152141), Food and Health Bureau, Hong Kong.

\section{Author contributions}

Minyan Zeng was involved in study design, collection and analysis of data, and draft writing. Qi Feng carried out study design, collection and analysis of data, and critical revision of manuscript. Ming Lu performed study design, funding acquisition, and critical revision of manuscript. Jun Zhou contributed toward study design, funding acquisition, and critical revision of manuscript. Zuyao Yang was involved in study conception and design, funding acquisition, collection and analysis of data, and critical revision of manuscript. Jinling Tang carried out study design, funding acquisition, and critical revision of manuscript. All authors contributed to data analysis, drafting and revising the article, gave final approval of the version to be published, and agree to be accountable for all aspects of the work.

\section{Disclosure}

The authors report no conflicts of interest in this work.

\section{References}

1. Siegel RL, Miller KD, Jemal A, Cancer Statistics, 2017. Ca-Cancer J Clin. 2017. 2017;67(1):7-30.

2. Bond-Smith G, Banga N, Hammond TM, Imber CJ. Pancreatic adenocarcinoma. BMJ. 2012;344(16):e2476.

3. Registry H.K.C. Hong Kong Cancer Statistics; 2015. Available from: http://www3.ha.org.hk/cancereg/pdf/overview/Summary\%20of\%20 CanStat\%202015.pdf. Accessed January 17, 2018.

4. Wilkowski R, Wolf M, Heinemann V. Primary advanced unresectable pancreatic cancer. Recent Results Cancer Res. 2008;177:79-93.

5. Chan SL, Chan ST, Chan EH, He ZX, Zx H. Systemic treatment for inoperable pancreatic adenocarcinoma: review and update. Chin $J$ Cancer. 2014;33(6):267-276.

6. Burris HA. 3rd., Moore MJ, Andersen J. Improvements in survival and clinical benefit with gemcitabine as first-line therapy for patients with advanced pancreas cancer: a randomized trial. J Clin Onco. 1997; 15(6):2403-2413.

7. Moore MJ, Goldstein D, Hamm J, et al. Erlotinib plus gemcitabine compared with gemcitabine alone in patients with advanced pancreatic cancer: a phase III trial of the National Cancer Institute of Canada Clinical Trials Group. J Clin Oncol. 2007;25(15):1960-1966.

8. von Hoff DD, Ervin T, Arena FP, et al. Increased survival in pancreatic cancer with nab-paclitaxel plus gemcitabine. N Engl J Med. 2013; 369(18):1691-1703.

9. Conroy T, Desseigne F, Ychou M, et al. FOLFIRINOX versus gemcitabine for metastatic pancreatic cancer. $N$ Engl J Med. 2011;364(19): $1817-1825$.

10. Aranda E, Manzano JL, Rivera F, et al. Phase II open-label study of erlotinib in combination with gemcitabine in unresectable and/or metastatic adenocarcinoma of the pancreas: relationship between skin rash and survival (Pantar study). Ann Oncol. 2012;23(7):1919-1925.

11. Beveridge RD, Alcolea V, Aparicio J, et al. Management of advanced pancreatic cancer with gemcitabine plus erlotinib: efficacy and safety results in clinical practice. JOP. 2014;15(1):19-24.
12. Petrelli F, Borgonovo K, Cabiddu M, Lonati V, Barni S. Relationship between skin rash and outcome in non-small-cell lung cancer patients treated with anti-EGFR tyrosine kinase inhibitors: a literature-based meta-analysis of 24 trials. Lung Cancer. 2012;78(1):8-15.

13. Soulieres D, Senzer NN, Vokes EE, Hidalgo M, Agarwala SS, Siu LL. Multicenter phase II study of erlotinib, an oral epidermal growth factor receptor tyrosine kinase inhibitor, in patients with recurrent or metastatic squamous cell cancer of the head and neck. J Clin Oncol. 2004; 22(1):77-85.

14. Klapdor R, Klapdor S, Bahlo M. Combination therapy with gemcitabine (GEM) and erlotinib (E) in exocrine pancreatic cancer under special reference to RASH and the tumour marker CA19-9. Anticancer Res. 2012;32(5):2191-2197.

15. Renouf DJ, Tang PA, Hedley D, et al. A phase II study of erlotinib in gemcitabine refractory advanced pancreatic cancer. Eur J Cancer. 2014;50(11):1909-1915.

16. Feliu J, Borrega P, León A, et al. Phase II study of a fixed dose-rate infusion of gemcitabine associated with erlotinib in advanced pancreatic cancer. Cancer Chemother Pharmacol. 2011;67(1):215-221.

17. Munoz Llarena A, Lopez-Vivanco G, Ruiz de Lobera A, et al. Gemcitabine (G) fixed-dose-rate infusion (FDR) plus erlotinib (E) in patients with advanced pancreatic cancer (APC). J Clin Onco. 2011; 29(4_suppl):304.

18. Park S, Chung MJ, Park JY, et al. Phase II trial of erlotinib plus gemcitabine chemotherapy in korean patients with advanced pancreatic cancer and prognostic factors for chemotherapeutic response. Gut Liver. 2013;7(5):611-615.

19. Oh DY, Lee KW, Lee KH, et al. A phase II trial of erlotinib in combination with gemcitabine and capecitabine in previously untreated metastatic/recurrent pancreatic cancer: combined analysis with translational research. Invest New Drugs. 2012;30(3):1164-1174.

20. Muñoz A, Azkona E, Iza EI, et al. Efficacy and safety of fixed-dose-rate infusions of gemcitabine plus erlotinib for advanced pancreatic cancer. $J$ Anal Oncol. 2015;4(1):44-51.

21. Parmar MK, Torri V, Stewart L. Extracting summary statistics to perform meta-analyses of the published literature for survival endpoints. Stat Med. 1998;17(24):2815-2834.

22. Wells G, Shea B, O'Connell D, et al. The Newcastle-Ottawa Scale (NOS) for Assessing the Quality of Non-Randomized Studies in MetaAnalysis. Available from: http://www.medicine.mcgill.ca/rtamblyn/ Readings $\% 5$ CThe $\% 20$ Newcastle $\% 20-\% 20$ Scale $\% 20$ for $\% 20$ assessing $\% 20$ the $\% 20$ quality $\% 20$ of $\% 20$ nonrandomised $\% 20$ studies $\% 20$ in\%20meta-analyses.pdf. Accessed January 17, 2018.

23. Zang J, Xiang C, He J. Synthesis of median survival time in metaanalysis. Epidemiology. 2013;24(2):337-338

24. Higgins JP, Thompson SG, Deeks JJ, Altman DG. Measuring inconsistency in meta-analyses. BMJ. 2003;327(7414):557-560.

25. GSe HJPT [homepage on the Internet]. Cochrane Handbook for Systematic Reviews of Interventions Version 5.1.0. The Cochrane Collaboration. 2011. Available from: www.handbook.cochrane.org. Accessed January 17, 2018.

26. Tang JL, Liu JL, Jly L. Misleading funnel plot for detection of bias in meta-analysis. J Clin Epidemiol. 2000;53(5):477-484.

27. Duval S, Tweedie R. Trim and fill: A simple funnel-plot-based method of testing and adjusting for publication bias in meta-analysis. Biometrics. 2000;56(2):455-463.

28. Wacker B, Nagrani T, Weinberg J, Witt K, Clark G, Cagnoni PJ. Correlation between development of rash and efficacy in patients treated with the epidermal growth factor receptor tyrosine kinase inhibitor erlotinib in two large phase III studies. Clin Cancer Res. 2007;13(13): 3913-3921.

29. Okusaka T, Furuse J, Funakoshi A, et al. Phase II study of erlotinib plus gemcitabine in Japanese patients with unresectable pancreatic cancer. Cancer Sci. 2011;102(2):425-431.

30. Stepanski EJ, Reyes C, Walker MS, et al. The association of rash severity with overall survival: findings from patients receiving erlotinib for pancreatic cancer in the community setting. Pancreas. 2013; 42(1):32-36. 
31. van Cutsem E, Vervenne WL, Bennouna J, et al. Phase III trial of bevacizumab in combination with gemcitabine and erlotinib in patients with metastatic pancreatic cancer. J Clin Oncol. 2009;27(13):2231-2237.

32. Vaccaro V, Bria E, Sperduti I, et al. First-line erlotinib and fixed dose-rate gemcitabine for advanced pancreatic cancer. World $J$ Gastroenterol. 2013;19(28):4511-4519.

33. Oettle H, Tessen H, Groschek M. Non-interventional study with Erlotinib/Gemcitabine in patients with metastatic pancreatic cancer. Onkologie Conference. 2013;36(Supplement 7):123.

34. Wang JP, Wu CY, Yeh YC, et al. Erlotinib is effective in pancreatic cancer with epidermal growth factor receptor mutations: a randomized, open-label, prospective trial. Oncotarget. 2015;6(20):18162-18173.

35. Irigoyen A, Gallego J, Guillén Ponce C, et al. Gemcitabine-erlotinib versus gemcitabine-erlotinib-capecitabine in the first-line treatment of patients with metastatic pancreatic cancer: Efficacy and safety results of a phase IIb randomised study from the Spanish TTD Collaborative Group. Eur J Cancer. 2017;75:73-82.

36. Irie $\mathrm{K}$, Ueno $\mathrm{M}$, Kobayashi $\mathrm{S}$, et al. The biomarkers of gemcitabine and erlotinib treatment in advanced pancreatic cancer. J Clin Oncol. 2014;32(3_suppl):358.

37. Cheng YJ, Bai CM, Zhang ZJ. Efficacy of Gemcitabine Combined with Erlotinib in Patients with Advanced Pancreatic Cancer. ActaCTA Academiae Medicinae Sinica. 2010;32(4):421-423.

38. Gu YH, Shu YQ, Huang PW, et al. Observation of Erlotinib (Tarceva) combined with gemcitabine in advanced pancreatic adenocarcinoma. Chin Clin Oncol. 2006;11(7):515-517.

39. Guo XD, Xu W, Sun CH. Clinical effect of Erlotinib plus gemcitabine in treatment of advanced pancreas cancer. Chin J of Clinical Rational Drug Use. 2012;5(2B):21-22.

40. Liu HB, Wu Y, Lv TF, et al. Skin rash could predict the response to EGFR tyrosine kinase inhibitor and the prognosis for patients with non-small cell lung cancer: a systematic review and meta-analysis. PLoS One. 2013;8(1):e55128.

41. Petrelli F, Borgonovo K, Barni S. The predictive role of skin rash with cetuximab and panitumumab in colorectal cancer patients: a systematic review and meta-analysis of published trials. Target Oncol. 2013;8(3): 173-181.
42. Mao C, Yang ZY, Hu XF, Chen Q, Tang JL. PIK3CA exon 20 mutations as a potential biomarker for resistance to anti-EGFR monoclonal antibodies in KRAS wild-type metastatic colorectal cancer: a systematic review and meta-analysis. Ann Oncol. 2012;23(6):1518-1525.

43. Fjällskog ML, Lejonklou MH, Oberg KE, Eriksson BK, Janson ET. Expression of molecular targets for tyrosine kinase receptor antagonists in malignant endocrine pancreatic tumors. Clin Cancer Res. 2003;9(4): 1469-1473.

44. Lacouture ME. Mechanisms of cutaneous toxicities to EGFR inhibitors. Nat Rev Cancer. 2006;6(10):803-812.

45. Jost M, Kari C, Rodeck U. The EGF receptor - an essential regulator of multiple epidermal functions. Eur J Dermatol. 2000;10(7):505-510.

46. Faller BA, Burtness B. Treatment of pancreatic cancer with epidermal growth factor receptor-targeted therapy. Biologics. 2009;3:419-428.

47. van Cutsem E, Li CP, Nowara E, et al. Dose escalation to rash for erlotinib plus gemcitabine for metastatic pancreatic cancer: the phase II RACHEL study. Br J Cancer. 2014;111(11):2067-2075.

48. Hidalgo M, Siu LL, Nemunaitis J, et al. Phase I and pharmacologic study of OSI-774, an epidermal growth factor receptor tyrosine kinase inhibitor, in patients with advanced solid malignancies. J Clin Oncol. 2001;19(13):3267-3279.

49. Goodin S. Erlotinib: optimizing therapy with predictors of response? Clin Cancer Res. 2006;12(10):2961-2963.

50. Eisenhauer EA, Therasse P, Bogaerts J, et al. New response evaluation criteria in solid tumours: revised RECIST guideline (version 1.1). Eur J Cancer. 2009;45(2):228-247.

51. Dhani N, Tu D, Sargent DJ, Seymour L, Moore MJ. Alternate endpoints for screening phase II studies. Clin Cancer Res. 2009;15(6): 1873-1882.

52. Lynch TJ, Kim ES, Eaby B, Garey J, West DP, Lacouture ME. Epidermal growth factor receptor inhibitor-associated cutaneous toxicities: an evolving paradigm in clinical management. Oncologist. 2007;12(5):610-621. 
Zeng et al

Dovepress

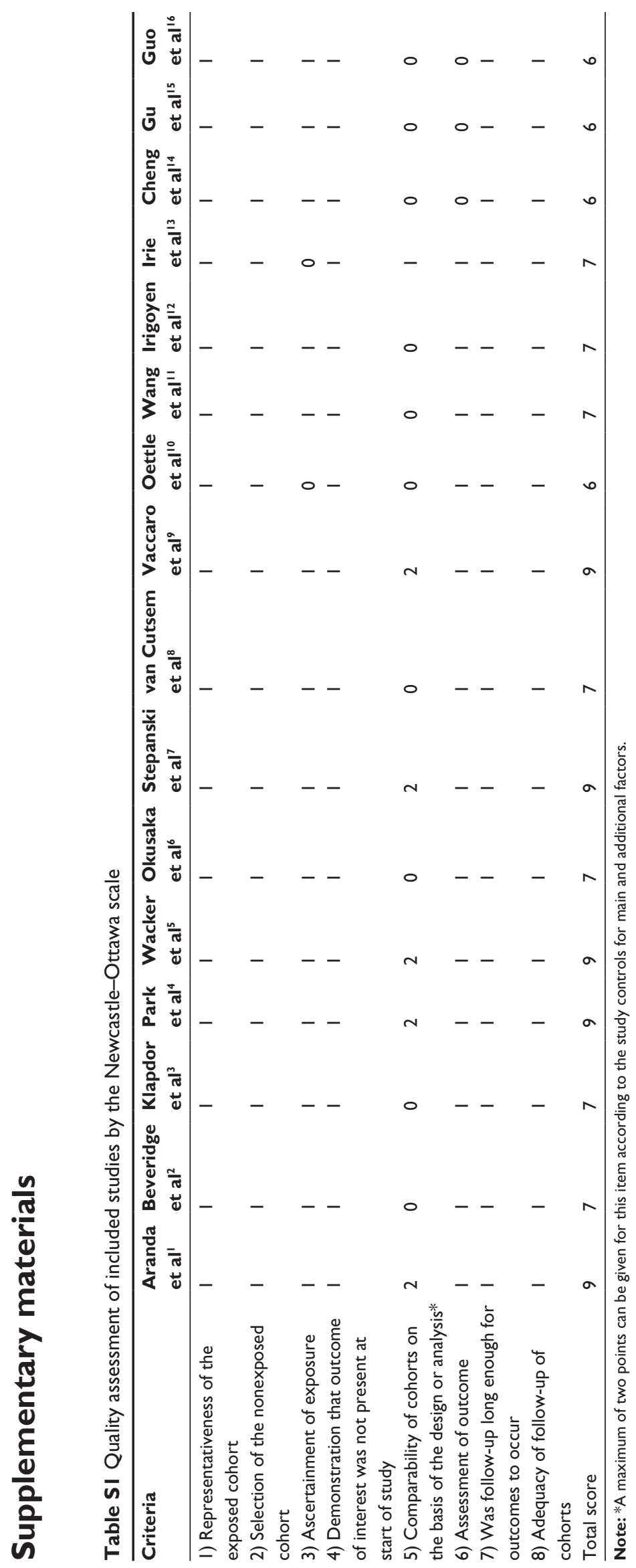

6644

submit your manuscript | www.dovepress.com

OncoTargets and Therapy 2018:1 I

Dovepress 
Table S2 Sensitivity analyses

\begin{tabular}{|c|c|c|c|}
\hline & Study & Patients & HR (95\% Cl) \\
\hline \multicolumn{4}{|l|}{ OS } \\
\hline Rash versus no rash (overall analysis) & 10 & $|, 42|$ & $0.57(0.50-0.64)$ \\
\hline Sensitivity analysis I & 9 & 1,366 & $0.57(0.50-0.65)$ \\
\hline Sensitivity analysis 2 & 6 & 999 & $0.55(0.48-0.64)$ \\
\hline Sensitivity analysis 3 & 9 & $\mathrm{I}, 04 \mathrm{I}$ & $0.54(0.47-0.62)$ \\
\hline Sensitivity analysis 4 & 2 & 105 & $0.32(0.15-0.66)$ \\
\hline Sensitivity analysis 5 & $\mathrm{I}$ & 46 & $0.20(0.05-0.7 \mathrm{I})$ \\
\hline Sensitivity analysis 6 & 9 & I,04I & $0.54(0.47-0.62)$ \\
\hline High-grade rash versus low-grade rash (overall analysis) & 4 & 487 & $0.63(0.49-0.80)$ \\
\hline Sensitivity analysis I & 3 & 317 & $0.60(0.44-0.83)$ \\
\hline Sensitivity analysis 4 & 2 & 321 & $0.59(0.43-0.80)$ \\
\hline \multicolumn{4}{|l|}{ PFS } \\
\hline Rash versus no rash (overall analysis) & 7 & 927 & $0.53(0.40-0.68)$ \\
\hline Sensitivity analysis 2 & 4 & 476 & $0.4 \mathrm{I}(0.26-0.65)$ \\
\hline Sensitivity analysis 4 & 2 & 115 & $0.22(0.08-0.60)$ \\
\hline High-grade rash versus low-grade rash (overall analysis) & 4 & 487 & $0.59(0.46-0.76)$ \\
\hline Sensitivity analysis I & 3 & 317 & $0.52(0.40-0.67)$ \\
\hline Sensitivity analysis 4 & $\mathrm{I}$ & 168 & $0.79(0.54-1.15)$ \\
\hline \multicolumn{4}{|l|}{ Objective response } \\
\hline Rash versus no rash (overall analysis) & 5 & 359 & $1.87(0.87-4.02)$ \\
\hline Sensitivity analysis 6 & 3 & 328 & $2.10(0.9 \mid-4.85)$ \\
\hline
\end{tabular}

Notes: Sensitivity analysis I: excluding the studies that recruited a small number of patients receiving an additional therapy during the treatment. Sensitivity analysis 2 : excluding the studies where HR could only be estimated from survival curves. Sensitivity analysis 3: excluding the study where HR could only be estimated with median survival time and $P$-value. Sensitivity analysis 4 : excluding the studies that did not control for any confounders to estimate HR. Sensitivity analysis 5 : excluding the studies that did not control for other confounders to estimate HR. Sensitivity analysis 6: excluding the studies with a high risk of bias.

Abbreviations: OS, overall survival; PFS, progression-free survival.

Table S3 Subgroup analyses

\begin{tabular}{|c|c|c|c|c|c|}
\hline PFS (rash versus no rash) & Studies & Patients & HR $(95 \% \mathrm{Cl})$ & Heterogeneity $\left(I^{2}\right), \%$ & $P$-value \\
\hline \multicolumn{6}{|l|}{ Ethnicity } \\
\hline Asian & 2 & 113 & $0.40(0.25-0.63)$ & 0.0 & 0.242 \\
\hline White & 4 & 560 & $0.53(0.44-0.64)$ & 56.2 & - \\
\hline \multicolumn{6}{|l|}{ ECOG 0-I } \\
\hline ECOG $0-1 \geq 80 \%$ & 5 & 812 & $0.60(0.52-0.70)$ & 49.9 & 0.006 \\
\hline ECOG $0-I<80 \%$ & 2 & 115 & $0.25(0.13-0.46)$ & 53.8 & - \\
\hline \multicolumn{6}{|c|}{ Prior chemotherapy treatment for pancreatic cancer } \\
\hline With previously treated patients & 2 & 314 & $0.70(0.56-0.87)$ & 68.1 & 0.036 \\
\hline With treatment-naive patients & 5 & 613 & $0.5 \mathrm{I}(0.43-0.6 \mathrm{I})$ & 54.2 & - \\
\hline
\end{tabular}

Abbreviations: PFS, progression-free survival; ECOG, Eastern Cooperative Oncology Group.

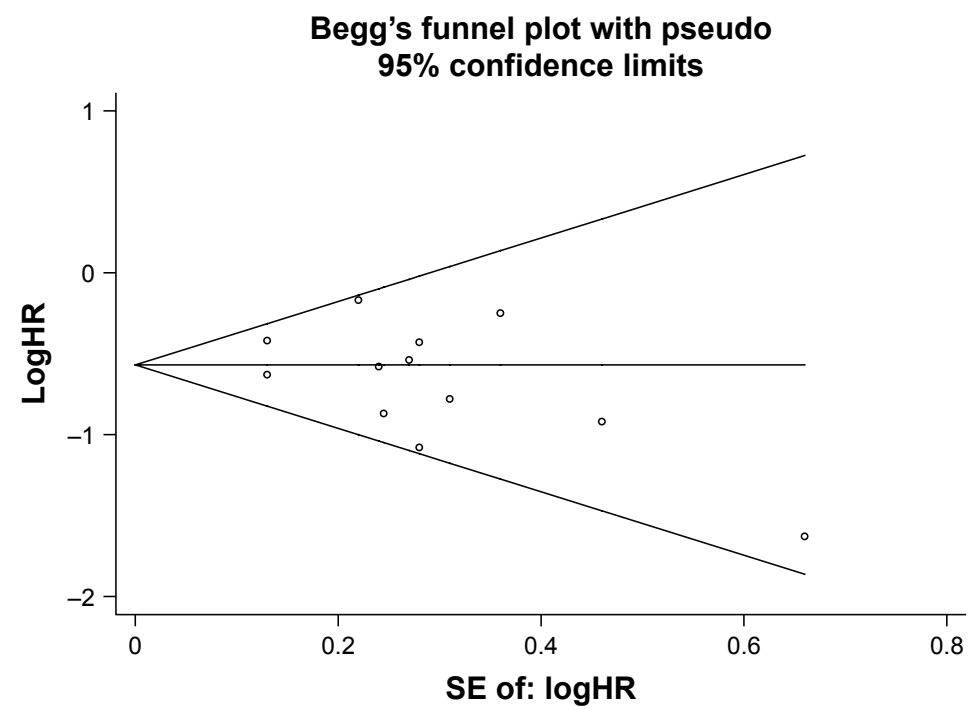

Figure SI Funnel plot based on the data of Figure 2A (HR for OS with versus without rash).

Abbreviations: OS, overall survival; $\mathrm{SE}$, standard error. 


\section{References}

1. Aranda E, Manzano JL, Rivera F, et al. Phase II open-label study of erlotinib in combination with gemcitabine in unresectable and/or metastatic adenocarcinoma of the pancreas: relationship between skin rash and survival (Pantar study). Ann Oncol. 2012;23(7):1919-1925.

2. Beveridge RD, Alcolea V, Aparicio J, et al. Management of advanced pancreatic cancer with gemcitabine plus erlotinib: efficacy and safety results in clinical practice. JOP. 2014;15(1):19-24.

3. Klapdor R, Klapdor S, Bahlo M. Combination therapy with gemcitabine (GEM) and erlotinib (E) in exocrine pancreatic cancer under special reference to RASH and the tumour marker CA19-9. Anticancer Res. 2012;32(5):2191-2197.

4. Park S, Chung MJ, Park JY, et al. Phase II trial of erlotinib plus gemcitabine chemotherapy in korean patients with advanced pancreatic cancer and prognostic factors for chemotherapeutic response. Gut Liver. 2013;7(5):611-615.

5. Wacker B, Nagrani T, Weinberg J, Witt K, Clark G, Cagnoni PJ. Correlation between development of rash and efficacy in patients treated with the epidermal growth factor receptor tyrosine kinase inhibitor erlotinib in two large phase III studies. Clin Cancer Res. 2007;13(13): 3913-3921.

6. Okusaka T, Furuse J, Funakoshi A, et al. Phase II study of erlotinib plus gemcitabine in Japanese patients with unresectable pancreatic cancer. Cancer Sci. 2011;102(2):425-431.

7. Stepanski EJ, Reyes C, Walker MS, et al. The association of rash severity with overall survival: findings from patients receiving erlotinib for pancreatic cancer in the community setting. Pancreas. 2013; 42(1):32-36.

8. van Cutsem E, Vervenne WL, Bennouna J, et al. Phase III trial of bevacizumab in combination with gemcitabine and erlotinib in patients with metastatic pancreatic cancer. J Clin Oncol. 2009;27(13):2231-2237.
9. Vaccaro V, Bria E, Sperduti I, et al. First-line erlotinib and fixed dose-rate gemcitabine for advanced pancreatic cancer. World $J$ Gastroenterol. 2013;19(28):4511-4519.

10. Oettle H, Tessen H, Groschek M. Non-interventional study with Erlotinib/Gemcitabine in patients with metastatic pancreatic cancer. Onkologie Conference. 2013;36(Supplement 7):123.

11. Wang JP, Wu CY, Yeh YC, et al. Erlotinib is effective in pancreatic cancer with epidermal growth factor receptor mutations: a randomized, open-label, prospective trial. Oncotarget. 2015;6(20):18162-18173.

12. Irigoyen A, Gallego J, Guillén Ponce C, et al. Gemcitabine-erlotinib versus gemcitabine-erlotinib-capecitabine in the first-line treatment of patients with metastatic pancreatic cancer: Efficacy and safety results of a phase IIb randomised study from the Spanish TTD Collaborative Group. Eur J Cancer. 2017;75:73-82.

13. Irie K, Ueno M, Kobayashi S, Gouda Y, Ohkawa S, Manabu M. The biomarkers of gemcitabine and erlotinib treatment in advanced pancreatic cancer. J Clin Oncol. 2014;32(3_suppl):358.

14. Cheng YJ, Bai CM, Zhang ZJ. Efficacy of Gemcitabine Combined with Erlotinib in Patients with Advanced Pancreatic Cancer. ActaCTA Academiae Medicinae Sinica. 2010;32(4):421-423.

15. Gu YH, Shu YQ, Huang PW, Zhu WY, Zhu CJ, Li W. Observation of Erlotinib (Tarceva) combined with gemcitabine in advanced pancreatic adenocarcinoma. Chin Clin Oncol. 2006;11(7):515-517.

16. Guo XD, Xu W, Sun CH. Clinical effect of Erlotinib plus gemcitabine in treatment of advanced pancreas cancer. Chin J of Clinical Rational Drug Use. 2012;5(2B):21-22.
OncoTargets and Therapy

\section{Publish your work in this journal}

OncoTargets and Therapy is an international, peer-reviewed, open access journal focusing on the pathological basis of all cancers, potential targets for therapy and treatment protocols employed to improve the management of cancer patients. The journal also focuses on the impact of management programs and new therapeutic agents and protocols on

\section{Dovepress}

patient perspectives such as quality of life, adherence and satisfaction. The manuscript management system is completely online and includes a very quick and fair peer-review system, which is all easy to use. Visit http://www.dovepress.com/testimonials.php to read real quotes from published authors. 